\title{
Flow States and Transitions of an Active Nematic in a Three-Dimensional Channel
}

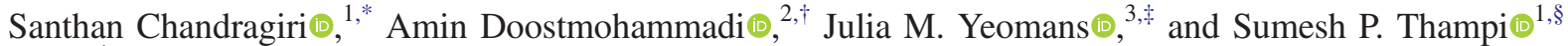 \\ ${ }^{1}$ Department of Chemical Engineering, Indian Institute of Technology Madras, Chennai 600036, India \\ ${ }^{2}$ Niels Bohr Institute, University of Copenhagen, Blegdamsvej 17, 2100 Copenhagen, Denmark \\ ${ }^{3}$ The Rudolf Peierls Centre for Theoretical Physics, Clarendon Laboratory, Parks Road, Oxford OX1 3PU, United Kingdom
}

(Received 28 April 2020; accepted 26 August 2020; published 30 September 2020)

\begin{abstract}
We use active nematohydrodynamics to study the flow of an active fluid in a 3D microchannel, finding a transition between active turbulence and regimes where there is a net flow along the channel. We show that the net flow is only possible if the active nematic is flow aligning and that, in agreement with experiments, the appearance of the net flow depends on the aspect ratio of the channel cross section. We explain our results in terms of when the hydrodynamic screening due to the channel walls allows the emergence of vortex rolls across the channel.
\end{abstract}

DOI: $10.1103 /$ PhysRevLett.125.148002

Spontaneous flow generation associated with collective dynamics in cell colonies [1,2], bacterial suspensions [3,4], and cytoskeletal elements, both in vivo [5] and in vitro $[6,7]$, has been of great scientific interest in recent years [8-11]. The continuous throughput of energy in these active systems manifests as active turbulence, a flow field characterized by spatiotemporally evolving fluid jets and vortices. Under the right conditions, such systems are capable of self-organization from chaotic flows into coherent flows: groups of active particles move together as a unit in a directed manner [2,12-14]. Coherent active flows are relevant to the formation of bacterial biofilms [15], wound healing [16], organ formation [17], and collective tumor invasion [18]. Beyond the biological implications, understanding how these self-sustained flows can be controlled and directed would prove to be a tremendous advance in microfluidics [19] where, conventionally, external flows are imposed for targeted drug delivery [20], for mixing in microreactors [21], or for pumping fluids at microscales [22]. An outstanding question is therefore how the chaotic motion of active matter can be translated into a selfsustained coherent flow.

Several lines of evidence demonstrate that geometric confinement can stabilize chaotic active motion into directed flows [2,12-14]. In two dimensions, theoretical analysis [23] predicts a transition from a quiescent fluid to a coherent, laminar flow through the channel as the channel height increases beyond a well-defined threshold for a given activity. The properties of this transition are reproduced quantitatively in experiments on fibroblast cells confined in 2D channels of varying width [2]. Beyond this linear instability, 2D experiments [13,24-27] and simulations [28-30] have shown that oscillating flows and vortex-lattice states also emerge by increasing the channel height before transitioning to active turbulence. Despite this extensive research on $2 \mathrm{D}$ systems, the understanding of 3D active flows is in its infancy [31-33]. Of particular interest, recent puzzling experiments show that the chaotic motion of a suspension of microtubules and molecular motors can be stabilized into a coherent flow in meter-long channels with a square cross section of any size but not in channels with a rectangular cross isosection [14]. (In this Letter, we will use the term "coherent flows" to refer to a state of broken symmetry along the channel length, whereby a net transport of fluid through the channel is obtained.) The maximum flow velocity reached about $10 \mathrm{~mm} \mathrm{~s}^{-1}$, which is comparable to the velocities of pump-driven flows routinely used in microfluidics. However, the physical mechanism behind the transition to a net flow and the reason for its dependence on the aspect ratio of the channel remain unexplained.

Here, to address these outstanding questions and to bridge the gap between 2D and 3D studies of confined active flows, we perform numerical simulations of active nematohydrodynamics. In a manner similar to the experimental conditions in $[14,24]$, the system is maintained above the isotropic-nematic transition temperature such that all the nematic ordering is activity-induced [34]. We find that an active fluid transitions from active turbulence to coherent flows as the aspect ratio of the confining channel is reduced. In addition, the simulations allow us to show that a necessary physical condition for such a crossover is that the system must be in the flow aligning regime, where the induced order aligns with the self-generated shear flows. We explain the underlying mechanism of the transition to coherent flows based on these observations.

Governing equations. - We consider a model, active nematic, incompressible fluid on a continuum scale, conserving mass and momentum [10,35]:

$$
\nabla \cdot \boldsymbol{u}=0 ; \quad \rho \frac{D \boldsymbol{u}}{D t}=\nabla \cdot \boldsymbol{\Pi},
$$


where $\rho, \boldsymbol{u}$, and $\boldsymbol{\Pi}$ represent the density, velocity, and stress field in the fluid, respectively, and $D / D t$ is the material derivative. Local orientational order is described using an order parameter $\boldsymbol{Q}=q(3 \boldsymbol{n n}-\boldsymbol{I}) / 2$, a second order traceless symmetric tensor field [36] where $q$ is the strength of the orientational order, $\boldsymbol{n}$ is the director field, and $\boldsymbol{I}$ is the identity tensor. The order parameter evolves according to [37-39]

$$
\frac{D Q}{D t}-S=\Gamma \boldsymbol{H}
$$

where $\quad \boldsymbol{S}=(\lambda \boldsymbol{E}+\boldsymbol{\Omega}) \cdot(\boldsymbol{Q}+\boldsymbol{I} / 3)+(\boldsymbol{Q}+\boldsymbol{I} / 3) \cdot(\lambda \boldsymbol{E}-$ $\boldsymbol{\Omega})-2 \lambda(\boldsymbol{Q}+\boldsymbol{I} / 3)(\boldsymbol{Q}: \nabla \boldsymbol{u})$ is the generalized advection term, $\Gamma$ is the rotational diffusivity, $\boldsymbol{H}=-A_{0}(1-\gamma / 3) \boldsymbol{Q}+$ $A_{0} \gamma\left(\boldsymbol{Q} \cdot \boldsymbol{Q}-(\boldsymbol{I} / 3) \boldsymbol{Q}^{2}\right)-A_{0} \gamma \boldsymbol{Q}^{2} \boldsymbol{Q}+K \nabla^{2} \boldsymbol{Q}$ is the molecular potential, $\boldsymbol{E}$ and $\boldsymbol{\Omega}$ are the symmetric and antisymmetric parts of the velocity gradient tensor, $A_{0}$ sets the scale for the free energy, $\gamma$ controls the temperature, and $K$ is an elastic constant determining the free energy cost of any variation in the order parameter. The alignment parameter $\lambda=$ $9 q \lambda_{1} /(3 q+4)$ determines the coupling between the velocity gradient and the orientational order. In the flow aligning regime $\lambda_{1}>1$, the director field aligns at a given angle to a shear flow, while in the flow tumbling regime $\lambda_{1}<1$, the director field rotates under shear.

The passive contributions to the stress $\boldsymbol{\Pi}$ are the Newtonian viscous stress, $\boldsymbol{\Pi}^{\text {viscous }}=2 \mu \boldsymbol{E}$ and an elastic stress, $\quad \boldsymbol{\Pi}^{\text {passive }}=-P \boldsymbol{I}+2 \lambda(\boldsymbol{Q}+\boldsymbol{I} / 3)(\boldsymbol{Q}: H)-\lambda \boldsymbol{H} \cdot(\boldsymbol{Q}+$ $\boldsymbol{I} / 3)-\lambda(\boldsymbol{Q}+\boldsymbol{I} / 3) \cdot \boldsymbol{H}-\nabla \boldsymbol{Q}:(K \nabla \boldsymbol{Q})+\boldsymbol{Q} \cdot \boldsymbol{H}-\boldsymbol{H} \cdot \boldsymbol{Q}$, where $\mu$ is the shear viscosity of the fluid and $P$ is the pressure. The activity of the fluid particles generates an active stress, $\boldsymbol{\Pi}^{\text {active }}=-\zeta \boldsymbol{Q}$ [40], where $\zeta$ describes the strength of the activity. Extensile active forcing, $\zeta>0$, is needed to give active turbulence in an isotropic phase [34].
Simulation details. - The equations of motion are solved using a lattice Boltzmann method for the mass and momentum conservation equations [Eq. (1)] and a method of lines for the order parameter evolution equation [Eq. (2)] $[38,41,42]$. It is not known how to map parameters in the continuum model to physical values, so we choose parameters in the range that reproduces the behavior of $2 \mathrm{D}$ microtubule and motor protein mixtures [24,43] and express all quantities in lattice units. The simulation domain [Fig. 1(a)] is a channel of length $L=128$, with height $h$ (shorter side) and width $w$ (longer side) between 8 and 96, $\Gamma=0.034, \quad K=0.03, \zeta=0.04, \quad \mu=0.6667$, $\rho=1$, and the free energy parameters are $A_{0}=0.1$ and $\gamma=2.6087$, corresponding to the isotropic state of the fluid $[29,38]$. Equating a typical length (size of the vortex) and a velocity scale from experiments $(100 \mu \mathrm{m}$ and $10 \mu \mathrm{m} / \mathrm{s}$ in [14]) and simulations (40 and 0.025 lattice units), our results correspond to flows in microchannels of width $20-240 \mu \mathrm{m}$ with velocity $0.12-36 \mu \mathrm{m} / \mathrm{s}$. We use no slip boundary conditions, and a no anchoring boundary condition is imposed on the orientational order parameter at the channel walls. Simulations are initialized with a stagnant fluid and a randomly oriented director field.

Results.-We begin by investigating the conditions that lead to a coherent (i.e., a net) flow along the channel. To this end, we define an order parameter

$$
\phi_{x}=\left|\left\langle\frac{u_{x}(x, y, z)}{|\boldsymbol{u}(x, y, z)|}\right\rangle_{x, y, z}\right|
$$

where $\langle\cdots\rangle_{j}$ denotes the average calculated along the $j$ th direction. If the flow is predominantly along the channel length $u_{x} \gg u_{y}, u_{z}$ everywhere in the channel, then $\phi_{x} \rightarrow 1$, whereas for turbulent flows, where there is no net transport of fluid along the channel, $\phi_{x} \rightarrow 0$.

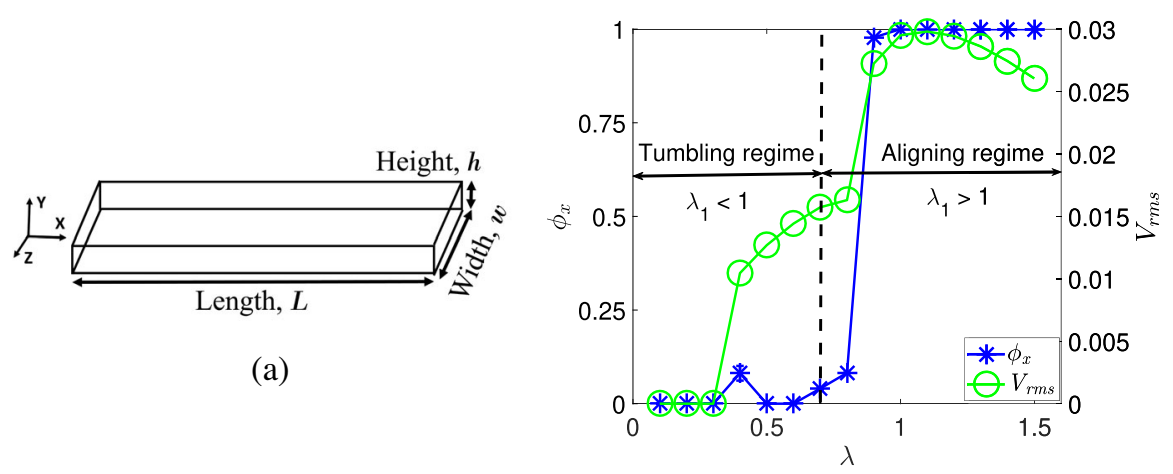

(b)

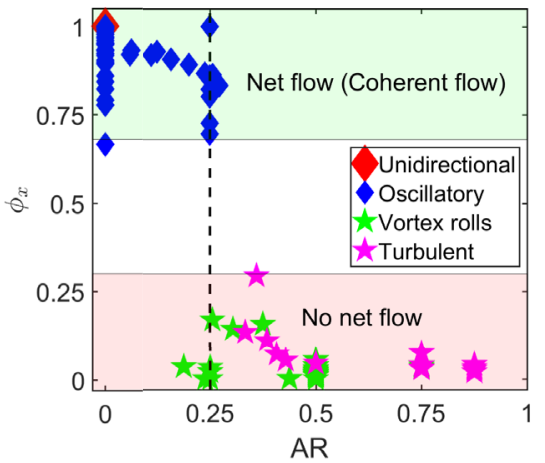

(c)

FIG. 1. Flow transition in an active nematic. (a) Schematic of the channel used in the simulations. (b) Variation of $\phi_{x}$ with $\lambda$ illustrating the turbulent $\left(\phi_{x} \approx 0\right)$ to coherent $\left(\phi_{x} \approx 1\right)$ flow transition. The corresponding root mean square velocity $V_{\text {rms }}$ is plotted on the secondary $y$ axis. The dashed line indicates the $\lambda$ corresponding to the tumbling-aligning transition in a simple shear flow. (c) Variation of $\phi_{x}$ with channel aspect ratio for flow aligning nematics $(\lambda=1.0)$. " $\diamond$ " indicates coherent (both unidirectional and oscillatory) and " $\star$ " indicates turbulent (and vortex roll) flows. The dashed line shows the approximate aspect ratio ("AR") at which the coherent to turbulent flow transition occurs. 
Figure 1(b) shows the variation of $\phi_{x}$ with the flow aligning parameter $\lambda$ for a square channel with $w=h=16$. It is evident that increasing $\lambda$ results in a sharp crossover from no net flow in the flow-tumbling regime to coherent flow when the fluid becomes flow aligning. We find that this crossover is robust to changing activity $\zeta$, orientational elasticity $K$, the dimensions of the channel $L$ and $w$ (in the range $500 \lesssim \zeta w^{2} / K \lesssim 10^{4}$ ), and the wall anchoring boundary conditions (see Supplemental Material [44]).

We next restrict ourselves to the flow aligning regime and, guided by the experiments [14], change the aspect ratio of the channel cross section. To quantify the aspect ratio, we define $\mathrm{AR}=(w-h) / w$. For square channels, $\mathrm{AR}=0$, whereas if $h$ and $w$ differ significantly, $\mathrm{AR} \rightarrow 1$.

Figure 1(c) summarizes the geometry dependence of the coherent to turbulent flow transition by measuring the flow order parameter $\phi_{x}$ as a function of the aspect ratio of the channel. Interestingly, there is a sharp transition between coherent flow states and active turbulence as the aspect ratio increases beyond $\sim 0.25$.

To understand this behavior, we use the simulations to more closely examine the details of the flow structure close to the transition from net flow to the active turbulent state. Near the transition point $\mathrm{AR} \approx 0.25$, neither a purely

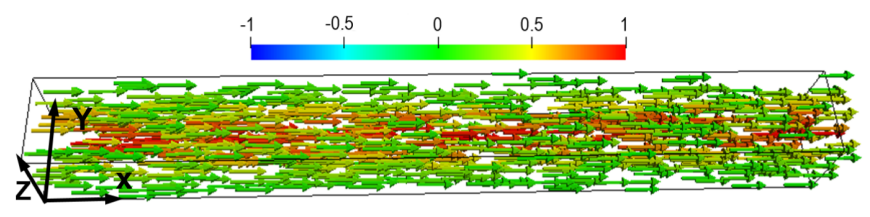

(a)

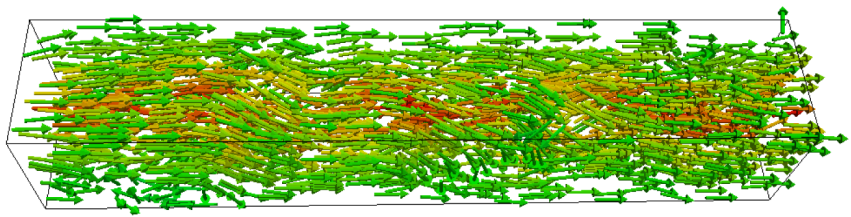

(c)

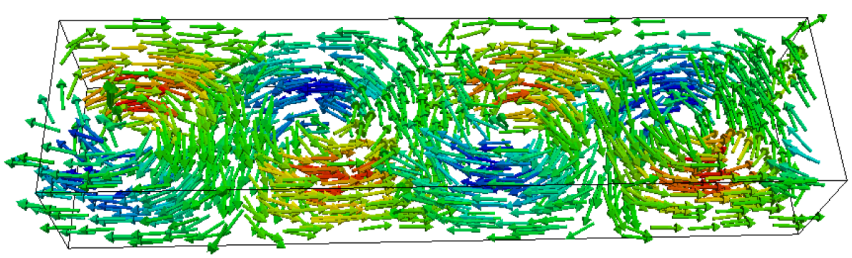

(e)

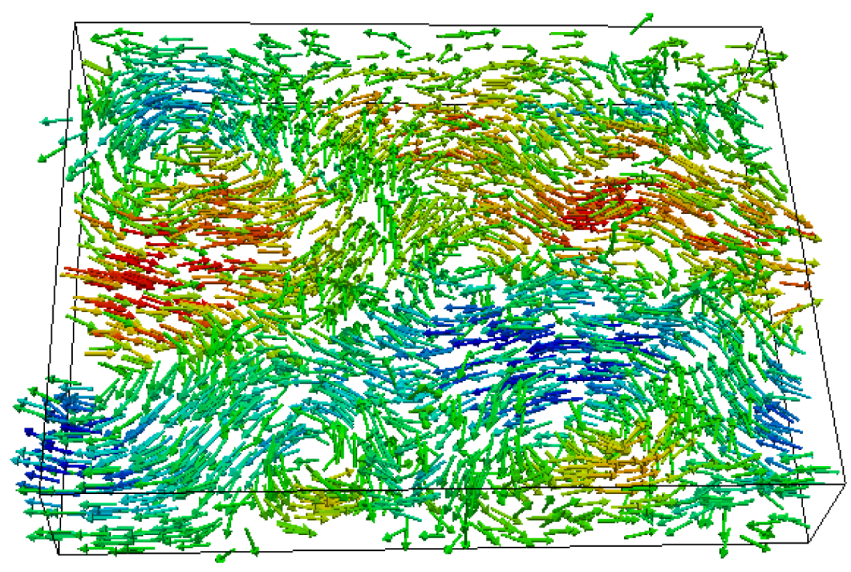

(g)

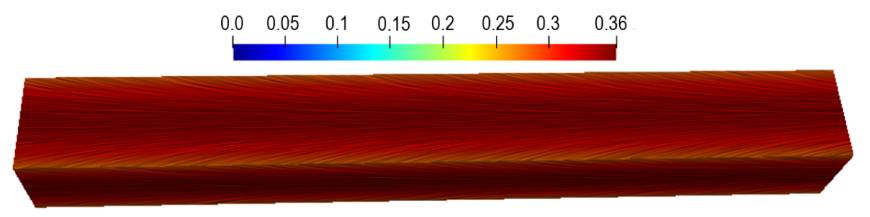

(b)

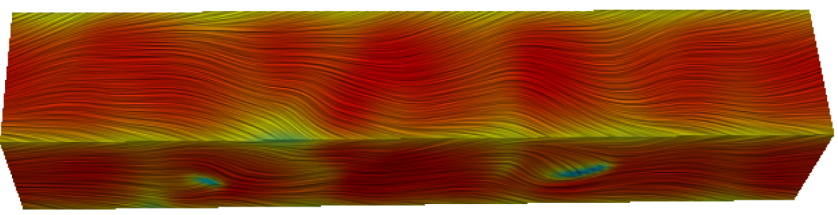

(d)

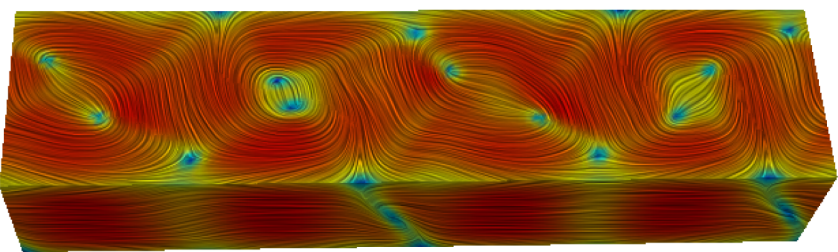

(f)

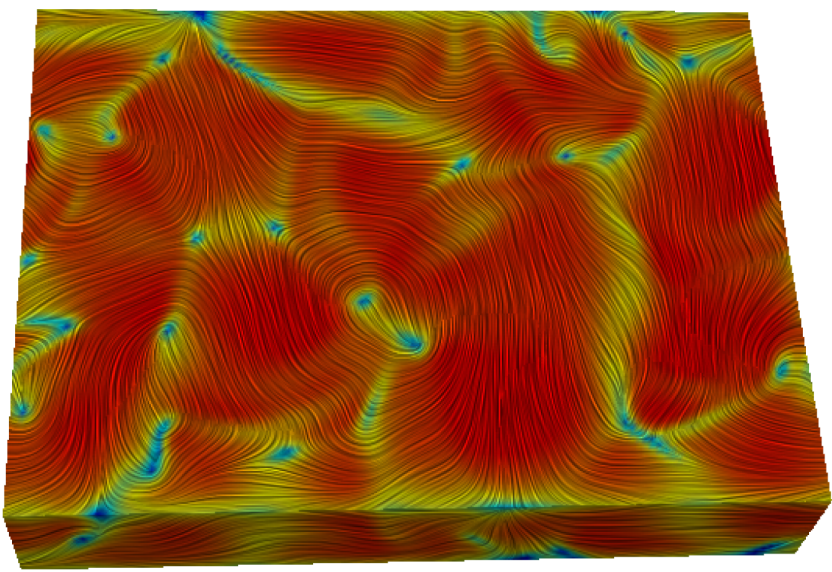

(h)

FIG. 2. Active nematic flow states in a 3D channel. (a),(b) Unidirectional $(h=w=16)$. (c),(d) Oscillatory ( $h=w=24)$. (e), (f) Lattice of vortex rolls $(h=24, w=32)$. (g),(h) Turbulent $(h=24, w=96)$ flow states. The left-hand column shows the velocity field color coded with normalized $u_{x}$. The right-hand column shows the director field, plotted as a line integral convolution, color coded with the scalar order parameter. Simulation parameters are $\lambda=1.0, K=0.013$, and $\zeta=0.022$. 
laminar flow nor a fully developed turbulent flow is observed. Instead, the active fluid can demonstrate oscillatory flow or a lattice of vortex rolls. The different flow states observed as the channel size is increased are shown in Fig. 2.

For very narrow channels, the active instability is suppressed and there is no flow. An increase in the channel size first leads to active flows that are completely unidirectional, with a velocity vector that only has a component along the channel length $\boldsymbol{u}=u_{x}(y, z) \hat{\boldsymbol{x}}$ [Fig. 2(a)]. For slightly wider channels, the flows can develop an oscillatory component and $u_{y} \neq u_{z} \neq 0$ [Fig. 2(c)]. However, the velocity along the channel remains the dominant component of the velocity field with its maximum value near the center line of the channel. Since $u_{x}$ is dominant in both unidirectional and oscillatory flows, these both manifest as coherent states that result in a net fluid transport through the channel.

Further increases in the size of the channel can, however, lead to flow configurations where the net flow is absent. The vortex-roll state is characterized by three-dimensional, counterrotating vortices located on a one-dimensional lattice along the length of the channel, as shown in Fig. 2(e). The axes of the vortices invariably lie along the smallest channel dimension ( $y$ direction). On any cross section perpendicular to the $y$ direction, the 3D flow appears similar to the dancing flows reported earlier in 2D simulations [28,29] and 2D confined microtubule and motor protein mixtures [26]. Regions with $q=0$ appear in the corresponding director field, indicating the presence of disclination lines [Fig. 2(f)]. These structures are dynamic and may form either in the bulk or near the walls. Finally, in large channels, we recover active turbulence, characterized by a spatiotemporally evolving director field [Figs. 2(g) and 2(h)] that results in a contortion of the disclination lines and their irregular spatial arrangement [31-33].

Mechanism.-We can now explain the disappearance of net flow as the aspect ratios of the channels increase. First, we note that, as a result of hydrodynamic screening, the vorticity correlation length in the channel $L_{\omega}$ is set by its smallest dimension $h$. Evidence for this is presented in Fig. 3(a), where we show that the vorticity correlation length $L_{\omega}$ (measured in the $x z$ plane at $y=h / 2$ ) tracks $h$ until the channel becomes too wide to screen the flows and the correlation length crosses over to its bulk value $L_{\omega}^{b}$. For comparison, Fig. 3(b) shows that there is no correlation between $L_{\omega}$ and the larger dimension of the channel cross section $w$. However, the structure of the flow is determined by $w$. If $w \lesssim L_{\omega}$, vortices cannot form, and there is a net flow. If, however, $w \gtrsim L_{\omega}$, the larger dimension of the channel cross section becomes available for the flow streamlines to turn, form vortices, and destroy the coherent net flow along the channel. This also explains why the vortex rolls are always ordered along the channel length with their axis along the shortest dimension of the channel. No analytical estimate for the exact value of the coefficient (a)

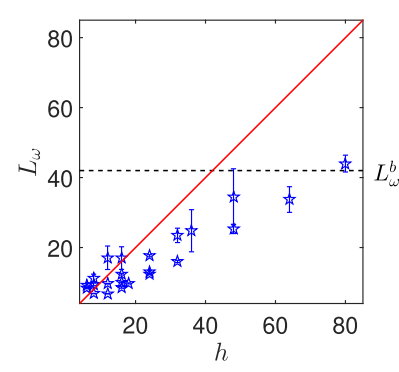

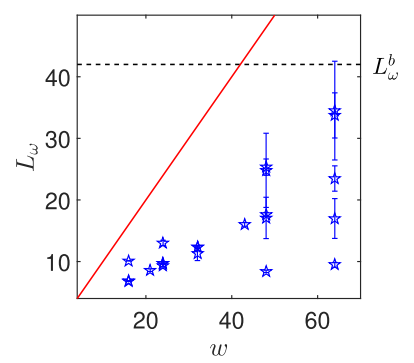

(b)
FIG. 3. Variation of vorticity correlation length against channel dimensions. (a) $L_{\omega}$ vs $h$ and (b) $L_{\omega}$ vs $w$. If $\boldsymbol{\omega}=\nabla \times \boldsymbol{u}$, then $L_{\omega}$ is determined as the distance $r$ at which the correlation function $\langle\boldsymbol{\omega}(r) \cdot \boldsymbol{\omega}(0)\rangle$ calculated in the $x z$ plane at $y=h / 2$ decays to zero. $L_{\omega}^{b}$ is the vorticity correlation length in the bulk calculated from bulk simulations. The solid line represents the line $x=y$ and the dashed line is drawn at $L_{\omega}=L_{\omega}^{b}$.

in these inequalities is available. However, numerical simulations of 2D confined active nematics [28] suggest that $w \sim 1.4 \times h$ when vortex rolls first form. This corresponds to $\mathrm{AR} \sim 0.28$. Note that the argument breaks down when both $w$ and $h$ are larger than the bulk correlation length $L_{\omega}^{b}$ : active turbulence will then destroy any net flow regardless of the aspect ratio.

Discussion.-Our results demonstrate that, in flow aligning, 3D, active microfluidics, the emergence of coherent net flow states is controlled by the aspect ratio of the channel cross section: coherent flows are possible in channels with cross sections that are close to isotropic but are destroyed in channels with larger aspect ratios. This can be explained by whether or not vortex rolls have room to form across the larger cross section dimension, thus destroying coherent flow. The length scale of the vortices is set by hydrodynamic screening controlled by the smaller dimension.

The aspect-ratio dependence agrees with recent experiments on microtubule-motor protein mixtures in microfluidic channels [14]. However, to more closely compare the results with the experimental system, the model in its current form requires a number of adjustments. First, here we assume a uniform density throughout the channel, while the experiments clearly indicate that layers of concentrated aligned microtubules can build up at the confining boundaries. Indeed, the experimental results were attributed to such surface ordering, but we show here that the aspectratio-induced flow transition does not require wetting of the channel walls by a nematic layer. Second, it is possible that free-slip velocity boundary conditions and weak-planar anchoring of the director field (see [44]) will be a more accurate representation of experiments as the microtubules appear to weakly align and slide freely at the boundaries $[25,26]$. We also show that the transition relies on the active fluid being flow aligning, thus identifying the microtubulemotor protein mixtures as belonging to this class of nematic fluids. 
A. D. acknowledges support from the Novo Nordisk Foundation (Grant No. NNF18SA0035142), Villum Fonden (Grant No. 29476), Danish Council for Independent Research, Natural Sciences (DFF-1171551001), and funding from the European Union's Horizon 2020 research and innovation program under the Marie Sklodowska-Curie grant agreement No. 847523 (INTERACTIONS).

Note added — Recently, we learned of the preprint by [45], which reports similar observations.

*Corresponding author.

ch15d413@smail.iitm.ac.in

${ }^{\dagger}$ Corresponding author.

doostmohammadi@nbi.ku.dk

*Corresponding author.

julia.yeomans@physics.ox.ac.uk

${ }^{\S}$ Corresponding author.

sumesh@iitm.ac.in

[1] T. B. Saw, A. Doostmohammadi, V. Nier, L. Kocgozlu, S. Thampi, Y. Toyama, P. Marcq, C. T. Lim, J. M. Yeomans, and B. Ladoux, Nature (London) 544, 212 (2017).

[2] G. Duclos, C. Blanch-Mercader, V. Yashunsky, G. Salbreux, J.-F. Joanny, J. Prost, and P. Silberzan, Nat. Phys. 14, 728 (2018).

[3] H. H. Wensink, J. Dunkel, S. Heidenreich, K. Drescher, R. E. Goldstein, H. Löwen, and J. M. Yeomans, Proc. Natl. Acad. Sci. U.S.A. 109, 14308 (2012).

[4] J. Dunkel, S. Heidenreich, K. Drescher, H. H. Wensink, M. Bär, and R. E. Goldstein, Phys. Rev. Lett. 110, 228102 (2013).

[5] A. Kumar, A. Maitra, M. Sumit, S. Ramaswamy, and G. Shivashankar, Sci. Rep. 4, 3781 (2014).

[6] K. Kruse, J.-F. Joanny, F. Jülicher, J. Prost, and K. Sekimoto, Phys. Rev. Lett. 92, 078101 (2004).

[7] V. Schaller, C. Weber, C. Semmrich, E. Frey, and A. R. Bausch, Nature (London) 467, 73 (2010).

[8] D. L. Koch and G. Subramanian, Annu. Rev. Fluid Mech. 43, 637 (2011).

[9] S. Ramaswamy, Annu. Rev. Condens. Matter Phys. 1, 323 (2010).

[10] M. C. Marchetti, J.-F. Joanny, S. Ramaswamy, T. B. Liverpool, J. Prost, M. Rao, and R. A. Simha, Rev. Mod. Phys. 85, 1143 (2013).

[11] G. Gompper, R. G. Winkler, T. Speck, A. Solon, C. Nardini, F. Peruani, H. Löwen, R. Golestanian, U. B. Kaupp, L. Alvarez et al., J. Phys. Condes. Matter 32, 193001 (2020).

[12] A. Bricard, J.-B. Caussin, N. Desreumaux, O. Dauchot, and D. Bartolo, Nature (London) 503, 95 (2013).

[13] H. Wioland, E. Lushi, and R. E. Goldstein, New J. Phys. 18, 075002 (2016).

[14] K.-T. Wu, J. B. Hishamunda, D. T. Chen, S. J. DeCamp, Y.-W. Chang, A. Fernández-Nieves, S. Fraden, and Z. Dogic, Science 355, eaal1979 (2017).

[15] J. C. Conrad and R. Poling-Skutvik, Annu. Rev. Chem. Biomol. Eng. 9, 175 (2018).
[16] M. Poujade, E. Grasland-Mongrain, A. Hertzog, J. Jouanneau, P. Chavrier, B. Ladoux, A. Buguin, and P. Silberzan, Proc. Natl. Acad. Sci. U.S.A. 104, 15988 (2007).

[17] R. McLennan, L. Dyson, K. W. Prather, J. A. Morrison, R. E. Baker, P. K. Maini, and P. M. Kulesa, Development 139, 2935 (2012).

[18] A. G. Clark and D. M. Vignjevic, Curr. Opin. Cell Biol. 36, 13 (2015).

[19] G. M. Whitesides, Nature (London) 442, 368 (2006).

[20] D. J. Beebe, G. A. Mensing, and G. M. Walker, Annu. Rev. Biomed. Eng. 4, 261 (2002).

[21] N.-T. Nguyen, S. T. Wereley, and S. A. M. Shaegh, Fundamentals and Applications of Microfluidics (Artech house, Norwood, 2019).

[22] D. J. Laser and J. G. Santiago, J. Micromech. Microeng. 14, R35 (2004).

[23] R. Voituriez, J.-F. Joanny, and J. Prost, Europhys. Lett. 70, 404 (2005).

[24] T. Sanchez, D. T. Chen, S. J. DeCamp, M. Heymann, and Z. Dogic, Nature (London) 491, 431 (2012).

[25] A. Opathalage, M. M. Norton, M. P. Juniper, B. Langeslay, S. A. Aghvami, S. Fraden, and Z. Dogic, Proc. Natl. Acad. Sci. U.S.A. 116, 4788 (2019).

[26] J. Hardoüin, R. Hughes, A. Doostmohammadi, J. Laurent, T. Lopez-Leon, J. M. Yeomans, J. Ignés-Mullol, and F. Sagués, Commun. Phys. 2, 1 (2019).

[27] K. Suzuki, M. Miyazaki, J. Takagi, T. Itabashi, and S. Ishiwata, Proc. Natl. Acad. Sci. U.S.A. 114, 2922 (2017).

[28] T. N. Shendruk, A. Doostmohammadi, K. Thijssen, and J. M. Yeomans, Soft Matter 13, 3853 (2017).

[29] S. Chandragiri, A. Doostmohammadi, J. M. Yeomans, and S. P. Thampi, Soft Matter 15, 1597 (2019).

[30] S. Chen, P. Gao, and T. Gao, J. Fluid Mech. 835, 393 (2018).

[31] T. N. Shendruk, K. Thijssen, J. M. Yeomans, and A. Doostmohammadi, Phys. Rev. E 98, 010601(R) (2018).

[32] J. Binysh, Ž. Kos, S. Čopar, M. Ravnik, and G. P. Alexander, Phys. Rev. Lett. 124, 088001 (2020).

[33] G. Duclos, R. Adkins, D. Banerjee, M. S. Peterson, M. Varghese, I. Kolvin, A. Baskaran, R. A. Pelcovits, T. R. Powers, A. Baskaran et al., Science 367, 1120 (2020).

[34] S. Santhosh, M. R. Nejad, A. Doostmohammadi, J. M. Yeomans, and S.P. Thampi, J. Stat. Phys. 180, 699 (2020).

[35] C. K. Batchelor and G. Batchelor, An Introduction to Fluid Dynamics, illustrated, reprint ed. (Cambridge University Press, Cambridge, England, 2000).

[36] P.-G. De Gennes and J. Prost, The Physics of Liquid Crystals (Oxford University Press, New York, 1995).

[37] L. Giomi, M. J. Bowick, X. Ma, and M. C. Marchetti, Phys. Rev. Lett. 110, 228101 (2013).

[38] D. Marenduzzo, E. Orlandini, M. E. Cates, and J. M. Yeomans, Phys. Rev. E 76, 031921 (2007).

[39] Ž. Kos, J. Aplinc, U. Mur, and M. Ravnik, in Flowing Matter (Springer, Cham, 2019), pp. 51-93.

[40] R. A. Simha and S. Ramaswamy, Phys. Rev. Lett. 89, 058101 (2002).

[41] A. Doostmohammadi, T. N. Shendruk, K. Thijssen, and J. M. Yeomans, Nat. Commun. 8, 15326 (2017). 
[42] J.-C. Desplat, I. Pagonabarraga, and P. Bladon, Comput. Phys. Commun. 134, 273 (2001)

[43] S. P. Thampi, R. Golestanian, and J. M. Yeomans, Phys. Rev. Lett. 111, 118101 (2013).
[44] See Supplemental Material at http://link.aps.org/ supplemental/10.1103/PhysRevLett.125.148002 for more data sets from simulations.

[45] M. Varghese et al., arXiv:2007.09277. 